\title{
A Patient-Centered Description of Severe Asthma: Patient Understanding Leading to Assessment for a Severe Asthma Referral (PULSAR)
}

\author{
Tonya A. Winders ${ }^{1} \cdot$ Andrew M. Wilson ${ }^{2,3,4} \cdot$ Monica J. Fletcher ${ }^{4,5} \cdot$ Anthony McGuinness $^{6} \cdot$ David B. Price $^{7,8,9}$
}

Published online: 17 July 2019

(c) The Author(s) 2019

\begin{abstract}
Background Although severe asthma can be life-threatening, many patients are unaware they have this condition. Objectives Patient Understanding Leading to Assessment for a Severe Asthma Referral (PULSAR) is a novel, multidisciplinary working group aiming to develop and disseminate a global, patient-centered description of severe asthma to improve patient understanding of severe asthma and effect a change in patient behavior whereby patients are encouraged to visit their healthcare professional, when appropriate.

Methods Current definitions from patient organization websites, asthma guidelines, and medication information for key asthma drugs were assessed and informed a multidisciplinary working group, convened to identify common concepts and terminology used to define severe asthma. A patient-centered description of severe asthma and patient checklist were drafted based on working-group discussions and reviewed by an external behavioral scientist for patient understanding and relevance. These were tested using an online US/Canadian survey.

Results The patient-centered description of severe asthma and patient checklist were reviewed and re-drafted by the authors. The text was simplified following the behavioral-scientist review. The survey $(n=153)$ included 105 patients with severe asthma. Of those with severe asthma, $92.2 \%$ of patients reported that the description was consistent with their experiences of severe asthma and $92.6 \%$ of patients reported that the PULSAR initiative would encourage them to visit their healthcare provider.

Conclusion A patient-centered description of severe asthma has been developed and tested using patients with severe asthma; this description will allow patients to assess whether they might have severe asthma and prompt them to visit their healthcare provider, if appropriate.
\end{abstract}

\section{Plain Language Summary}

Severe asthma is a serious form of asthma. It can be harmful to your health and affect the way you live your life. Some patients do not know that they have severe asthma or visit their doctor and ask for help. A new group, called Patient Understanding Leading to Assessment for a Severe Asthma Referral (PULSAR), would like to help patients understand their asthma

Electronic supplementary material The online version of this article (https://doi.org/10.1007/s40271-019-00371-0) contains supplementary material, which is available to authorized users.

Tonya A. Winders

twinders@AllergyAsthmaNetwork.org

1 Global Allergy Asthma Patient Platform (GAAPP), Vienna, Austria

2 University of East Anglia, Norwich, UK

3 Norfolk and Norwich University Hospital, Norwich, UK

4 Asthma UK Centre for Applied Research, Usher Institute, The University of Edinburgh, Edinburgh, UK
5 Global Respiratory Franchise, GlaxoSmithKline, Brentford, London, UK

6 Patient Representative, Birmingham, UK

7 Division of Applied Health Sciences, Centre of Academic Primary Care, University of Aberdeen, Aberdeen, UK

8 Observational and Pragmatic Research Institute, Singapore, Singapore

9 Optimum Patient Care Ltd, Oakington, Cambridge, UK 
symptoms. They have developed a description of severe asthma and a checklist. These may help patients decide if their symptoms require a visit to the doctor.

The PULSAR description and checklist were developed in four parts. Part 1 looked at if patients and doctors/nurses defined severe asthma in the same way. Results showed that patients defined severe asthma using symptoms and doctors defined severe asthma using treatments. In Part 2, patients, patient advocacy group members, nurses, doctors, specialists, and a scientist talked about the ways severe asthma were described in Part 1 . The group agreed on a set of words to describe severe asthma. These words were then used in the PULSAR description and checklist. In Part 3, a behavioral scientist reviewed the PULSAR description and checklist. They said that simple language would make it easy to understand. In Part 4, patients with severe asthma were asked what they thought about the description and checklist using an online survey. The survey showed that almost all patients understood the description and checklist. Many patients said that the description and checklist encouraged them to see a doctor.

A new description of severe asthma and checklist have been developed by PULSAR. Testing shows that they should encourage patients to visit their doctor when needed. This may help patients understand their symptoms and help doctors make the correct diagnosis. This should help patients get the support and treatment they need.

\section{Key Points for Decision Makers}

The new Patient Understanding Leading to Assessment for a Severe Asthma Referral (PULSAR) description of severe asthma could improve patient understanding of the condition and allow patients to assess whether their asthma may be severe.

The PULSAR description should encourage patients to visit their primary care physician, who may refer them for specialist investigations, if appropriate.

\section{Introduction}

Severe asthma is a life-threatening and debilitating condition that is associated with significant disease burden that can affect health-related quality of life (HRQoL) [1-3]. However, many patients with severe asthma are often unaware that their asthma is severe, possibly due to the different definitions provided by patient websites [4-17] or because many patients often underestimate their condition, believing that their symptoms are acceptable [18]. Consequently, some patients do not visit their primary care provider (PCP) and do not receive the appropriate level of care [18]. In addition, healthcare professionals (HCPs), who are faced with multiple asthma guidelines that differ in their approach to defining severe asthma [2, 3, 19-25], may not use language that patients understand. This may complicate the diagnosis and management of the disease and potentially impact on the number of patients referred to specialists and their subsequent access to appropriate therapies [26]. Therefore, there is a need to improve patient understanding of severe asthma and effect a change in patient behavior whereby patients are appropriately encouraged and have more confidence to visit and discuss their disease with their PCP; the PCP may then make a referral to a specialist [3]. Patient Understanding Leading to Assessment for a Severe Asthma Referral (PULSAR) is a new multidisciplinary initiative that aims to develop and disseminate a global patient-centered description of severe asthma for use by patients. This PULSAR description will allow patients to assess whether they might have severe asthma and prompt them to visit their PCP, if appropriate. This article describes the development of the PULSAR description. This was based on four distinct phases, which involved an assessment of current severe asthma definitions (Part 1); a discussion on key concepts and terminology in severe asthma by a multidisciplinary working group (Part 2); an evaluation by an external behavioral scientist (Part 3); and, finally, a patient survey to sense check the description (Part 4).

\section{Part 1: Assessment of Current Severe Asthma Definitions}

To identify the current definitions and terminology available to both English-speaking patients and HCPs, patient organization websites, current global and regional asthma guidelines including regulatory body websites, and medication information for key asthma drugs were searched and analyzed in April 2018. Further details are summarized in Additional File 1 of the electronic supplementary material. Overall, patient advocacy group websites defined severe asthma using symptoms $[8,12-15]$, possibly due to their ease of understanding and relevance to patients. Specific phrases used included 'difficulty breathing' [8], 'uncontrolled' [12], 'worsening of symptoms' [13], 'life-threatening' [8], 'symptoms during the day' [14], 'night-time awakenings every night' [14], or 'symptoms that do not improve or remain despite usual/preventer medications' [12, 15]. Additional 
phrases used by patient websites to define severe asthma included 'inability to take part in activities' [8], 'quality of life' [9], and 'level of medical care' [8]. The Severe Asthma Foundation and Asthma Canada websites defined severe asthma using a treatment-based approach, based on highdose treatment and a number of additional controllers [8, 16]. Only Asthma UK mentioned that severe asthma can only be diagnosed once poor adherence, inadequate technique, and other causes and triggers for symptoms have been ruled out [12]. Interestingly, several of the patient group websites did not include a definition of severe asthma [4-7, $10,11,27]$.

In contrast, most asthma guidelines base their definitions on treatment failure according to clinical criteria, despite receiving high-intensity treatment (medium- or high-dose controller medications) [2, 3, 19, 20, 22-25] or when high-dose treatment is reduced [3]. The sudden worsening of symptoms (asthma attacks/flare-ups/exacerbations) is considered by the Global Initiative for Asthma (GINA), the National Institutes of Health (NIH), and the Australian, Japanese, and World Health Organization (WHO) guidelines as part of their definition of severe asthma [2, $20,23,25]$, while other guidelines refer to these events as an acute episode that is separate to disease severity [21]. Other components considered in the guidelines include lung function [2, 21-23, 25], level of medical care [19], clinical investigations (e.g., eosinophil counts, allergy testing) [2, 3, 20-22], asthma phenotypes [2, 3, 19-22], and patient history of near fatal asthma or inpatient admissions [21]. Although some guidelines do not differentiate between severe and difficult-to-control or uncontrolled asthma [19, 25], many have specified that asthma is not considered severe if it can be controlled following correction/treatment of poor adherence, inappropriate or incorrect use of medications, environmental triggers, and comorbidities [2, 3, 21, 22, 24]. Of note, the WHO and NIH guidelines include a definition of severe asthma for untreated populations [20,25]. These definitions have been specified to either consider patient populations in developing countries, who do not have access to treatments such as inhaled corticosteroids (ICS) [20], or patients at first presentation [25]; however, these patients might be considered by some guidelines as having mild-moderate asthma if symptoms improve upon treatment [20]. It is important to note that there has been a shift in the medical definition of severe asthma over time. In the past, definitions varied markedly, often with separate definitions for patients before and during treatment. However, since the European Respiratory Society/American Thoracic Society (ERS/ATS) Task Force was established in 2009, with the resulting publication of the ERS/ATS Guidelines on Severe Asthma in 2014 [22], there has been general agreement that severe asthma is asthma that remains uncontrolled (in terms of symptoms, lung function, and/or exacerbations) despite high-dose treatment or that worsens when treatment is stepped down.

Overall, the labeling information (e.g., Prescribing Information, Summary of Product Characteristics, etc.) provided for severe asthma drugs does not specifically define severe asthma. However, the labeling information for the ICS/longacting- $\beta_{2}$-agonist (LABA) class indicates that these drugs can be given to patients not adequately controlled on ICS or whose treatment requires ICS/LABA [28], consistent with the treatment-based approach described by the asthma guidelines. Likewise, the injectable biologics are indicated for patients inadequately controlled on high-dose inhalers plus an additional controller medication [29-32], although they differ in that they are only indicated for specific forms of asthma (e.g., eosinophilic and allergic asthma) [29-32]. Therefore, the labeling information does not make it clear these drugs are indicated for severe asthma.

\section{Part 2: Identification of Key Terminology and Concepts for the PULSAR Description}

A multidisciplinary working group convened in June 2018 to discuss the current asthma definitions and produce a draft patient-centered description of severe asthma. This group included two patients with severe asthma, four patient advocacy group members, two nursing specialists, one PCP, three respiratory specialists, and one clinical scientist from GSK. Attendees were from a variety of countries, including Australia, Belgium, Canada, France, United Kingdom (UK), and United States of America (USA). During the working group, attendees reviewed the concepts and terminology used by the patient advocacy group websites, asthma guidelines, and medication information guidelines and, agreed on common concepts (treatments, symptoms, activity levels, and emotions) used to describe severe asthma. Attendees also voted on preferred, patient-friendly terms for inclusion/ exclusion in the description. A patient-centered description of severe asthma was drafted based on these discussions, which underwent subsequent reviews and re-drafting by the authors (detailed below).

\subsection{Concepts}

\subsubsection{Symptoms}

The most significant severe symptoms of asthma described by the patients included the inability to walk up a flight of stairs, persistent wheezing, the proverbial 'elephant on your chest', and recurrent chest infections. Patients noted that these symptoms can occur daily and never go away. As patients with milder forms of asthma may also experience these symptoms (especially during an asthma attack/ 
flare-up), the working group decided these symptoms could not be used to differentiate between severe and milder forms of asthma. Instead, to ensure that the concept was specific to severe asthma, symptoms were included if they were severe and frequently worsened (asthma attacks/flare-up), or required treatment with steroid tablets, use of emergency services, or hospitalization.

\subsubsection{Activities}

The patients in the working group expressed that they experience persistently reduced activity levels, meaning that they cannot do the things they want to do or require support from others to help them with everyday activities. Their reduced activity levels were related to their severe asthma symptoms, which affect their ability to maintain a conversation or activity, attendance at work, capacity to apply for their preferred job, exercise (patients cannot exercise as they would like or as much as their peers), and intimacy. As patients emphasized that they had to make financial, social, and lifestyle compromises to accommodate their asthma symptoms, more so than in milder forms of asthma, the PULSAR description included the compromises that patients with severe asthma are often forced to make.

\subsubsection{Emotions}

The patients discussed how living with severe asthma affected their lives in terms of their feelings. Common experiences included fear, frustration, fatigue, depression, helplessness and embarrassment, and that nothing seems to work in terms of treatment. The patients and patient advocacy group members recommended that the PULSAR description should encompass patient emotions as this concept is rarely covered by other definitions. However, as some of these emotions are experienced by patients with milder forms of asthma, the PULSAR description was drafted to encompass the overall impact of these emotions on patients' lives by stating that severe asthma can affect a patient's entire life. This is likely to be specific to severe asthma.

\subsubsection{Treatment}

The working group discussed the different treatment options for asthma and how they relate to severity, specifically controller and reliever medications as well as oral corticosteroid (OCS) use. The HCPs felt that patients with severe asthma often have symptoms despite receiving multiple moderate-/high-dose controller medications or if they reduce high-intensity treatment. However, the patients and patient advocacy group members were not sure whether this concept was patient-friendly and felt that it was possibly more relevant to HCPs. To ensure that the PULSAR description was specific to severe asthma, the working group decided that the PULSAR description should state that patients with severe asthma experience symptoms despite taking multiple high-dose medications as agreed. Reliever medications were mostly relevant to the patients as they noticed that they require them frequently (more than twice per week as recommended by GINA 2018 [2]), either in the form of inhalers or nebulizers, to gain relief from symptoms. However, as HCPs felt that the use of relievers is relevant to both patient groups with uncontrolled and/or severe asthma, reliever use could not be used to differentiate between these two patient groups. Therefore, this concept was not included in the PULSAR description to avoid confusion. As both patients mentioned that they often ( $\geq 2$ times a year) require courses of steroid tablets when they experience asthma attacks/flare-ups, the use of steroid tablets was included. Although the patients also described the unwanted side effects associated with OCS (e.g., weight gain, irritability, changes in mood, moon face, insomnia), which can discourage patients from taking their medications as prescribed, this concept was not included in the PULSAR description as it could be relevant to patients with milder forms of asthma.

\subsubsection{Call-to-Action}

The patient advocacy group attendees suggested including a 'call-to-action' within the PULSAR description to provide patients with advice on when to visit their PCP and what to expect in terms of secondary care activities. However, there was a difference between the HCPs and patients/patient advocacy group members on the language used to describe referrals. For example, patients and patient advocacy group members suggested that if patients think that they might have severe asthma they should visit their PCP and ask to be referred to a specialist. However, HCPs suggested that advising patients to ask for a referral may be too strong. To accommodate the views of both the patients/patient advocacy group members and HCPs, the PULSAR description recommended that patients should visit their PCP and discuss the possibility of a referral to a specialist.

\subsection{Terminology}

Terms were considered suitable for inclusion in the PULSAR description if they were patient-friendly and relevant for severe asthma. Terms were excluded if they could be interpreted differently by patients and HCPs or if they implied blame to either party, as this may lead to patients feeling guilty and not seeking help (Table 1). In instances where terms were appropriate for severe asthma but were not specifically patientfriendly, the group discussed alternative phrasing so that they could be included in the PULSAR description. For example, many attendees thought that 'exacerbations' was often 
Table 1 Terminology to be excluded from the patient-centered description

\begin{tabular}{|c|c|}
\hline Category & Terms \\
\hline Disease descriptor & $\begin{array}{l}\text { Uncontrolled } \\
\text { Phenotype } \\
\text { Reversibility } \\
\text { Chronic } \\
\text { Acute severe } \\
\text { Lack of control } \\
\text { A state of permanency } \\
\text { Treatment-resistant } \\
\text { Refractory }\end{array}$ \\
\hline Impacts & Shakes \\
\hline Exacerbations & Inpatient/outpatient visits \\
\hline Treatments & $\begin{array}{l}\text { Monoclonal antibodies } \\
\text { Bronchodilator } \\
\text { Biologics } \\
\text { Injections } \\
\text { Bronchial thermoplasty } \\
\text { Leukotriene receptor } \\
\text { antagonists } \\
\text { Oral } \\
\text { Color of inhaler (e.g., } \\
\text { brown or blue inhalers) }\end{array}$ \\
\hline Confounders & $\begin{array}{l}\text { Non-adherence } \\
\text { Confounders } \\
\text { Compliance } \\
\text { Diligence } \\
\text { Harmful }^{\mathrm{a}}\end{array}$ \\
\hline Clinical investigations & Spirometry \\
\hline
\end{tabular}

${ }^{\text {a }}$ Taken from Pavord et al. [43] regarding patient behavior and environment. Aspects include unscheduled visits, failure to attend appointments, poor adherence, smoking, allergenic environment, denial, depression, and disorganization

misunderstood by patients and decided that 'asthma-flares' or 'flare-ups' (North American terms) or 'asthma attacks' (UK term) would be easier to understand by patients. Similarly, many thought that the term 'oral corticosteroids' may be difficult to interpret by patients, so 'steroid tablets' was suggested as an alternative. When discussing if patients have taken their medications as prescribed, it was suggested that patients may feel blamed for not following their PCP's advice. Therefore, the patients requested that the phrase 'as agreed' be included as an alternative to 'as prescribed' to remove any inference of blame and to demonstrate that it was a mutual agreement between the patient and PCP. Other aspects of the PULSAR description discussed by the group were whether to define uncontrolled asthma and whether to use the term 'life-threatening'. The group decided not to describe the differences between severe and uncontrolled asthma in the PULSAR description to avoid confusion for the patient, as ultimately the description was designed to get the patient to the doctor. The term 'life-threatening' was kept as requested by the patients, as it encompasses the magnitude of the condition from the patient's perspective. To allow for the PULSAR description to be relevant globally, US- and UK-friendly terms were used; general practitioner (GP; UK term) and PCP (North American term) were both used, and emergency services was used instead of Emergency Room (North American term) and Accident and Emergency (UK term).

\subsection{Patient Materials}

Two different patient materials were proposed to accompany the PULSAR description and assist patients with assessing whether they should visit their PCP for their condition. However, the authors decided to focus on one of the patient materials, a patient checklist, as a checkbox system provided an easy means for patients to assess their condition. The checklist delineates characteristics of severe asthma into red and yellow characteristics, where red characteristics indicate clear signals of severe asthma and yellow describe characteristics that are both concerning and suggestive of severe asthma. Initially, 'red flags' and 'risk factors' were considered instead of red and yellow characteristics, but these were deemed to be confusing for patients. All characteristics were included based on the working group discussions described above.

\section{Part 3: Behavioral Scientist}

The revised PULSAR description and checklist were reviewed by an external behavioral scientist and certified asthma educator to gain feedback on whether they would be understandable by patients and if they could effect a change in patient behavior. The review identified that both the PULSAR description and checklist were appropriate for patients with severe asthma but that they could be simplified to improve their accessibility to patients with a low reading and health literacy age. It was also recommended that the language should be more compelling, forthright, persuasive, personal, and globally relevant.

Upon recommendation by the behavioral scientist, the checklist was simplified to a shopping-list design and the items included on the list were referred to as 'signs'. These signs were listed as either 'clear signs of severe asthma' (red) or 'concerning signs of severe asthma' (yellow). The language was simplified to a reading level of around 14 years old (Flesch-Kincaid Grade Level 8), written in the first person and modified so that it would motivate patients to visit their PCP.

\section{Part 4: Survey}

The final PULSAR description was reviewed by adult patients who self-reported as having severe asthma through the Allergy Asthma Network (Fig. 1). Further details of 
the survey process are included in Additional File 1 of the electronic supplementary material. Of the 153 patients who initiated the survey, $75.2 \%$ were female and $62.1 \%$ were 35-64 years of age versus 25-35 years (16.3\%), over 65 years $(12.4 \%)$ or $18-24$ years of age $(9.2 \%)$. Most $(92 / 152 ; 60.5 \%)$ patients reported having asthma for $15+$ years, while others reported having asthma for $0-2$ years $(17 / 152 ; 11.2 \%), 3-5$ years $(8 / 152 ; 5.3 \%)$, and $6-15$ years $(35 / 152 ; 20.0 \%)$. Of those who had been told by their doctor that they had severe asthma (105/153; 68.6\%), 101/104 (96.2\%) patients reported that they understood the PULSAR description and 95/103 (92.2\%) patients reported that the description was consistent with their own experiences of severe asthma. Consistent with the description, 63/105 $(60.0 \%)$ patients felt that their experience of asthma was potentially life-threatening and 86/104 (82.7\%) felt like asthma forced them to make unwanted changes to their daily lives. The terminology used within the description, including 'multiple high-dose inhalers', 'steroid tablets' and 'asthma attacks/flare-ups' were reported as being understood by $96.2-98.1 \%$ of patients. Interestingly, slightly more $(64.8 \%)$ patients preferred the term 'flareups' versus 'asthma attacks', which may be due to the survey being conducted in the US where the term 'flare-ups' is commonly used. A total of 100/105 (95.2\%) patients reported that they understood the PULSAR checklist and of those who did not, one patient reported it was too long and one reported it was difficult to complete. The remaining patients did not provide a reason. Overall, as most ( $n=87 / 94 ; 92.6 \%$ ) patients reported that the PULSAR description and checklist would encourage them to see their PCP about their asthma, no further changes were made to the terminology or concepts included in either.

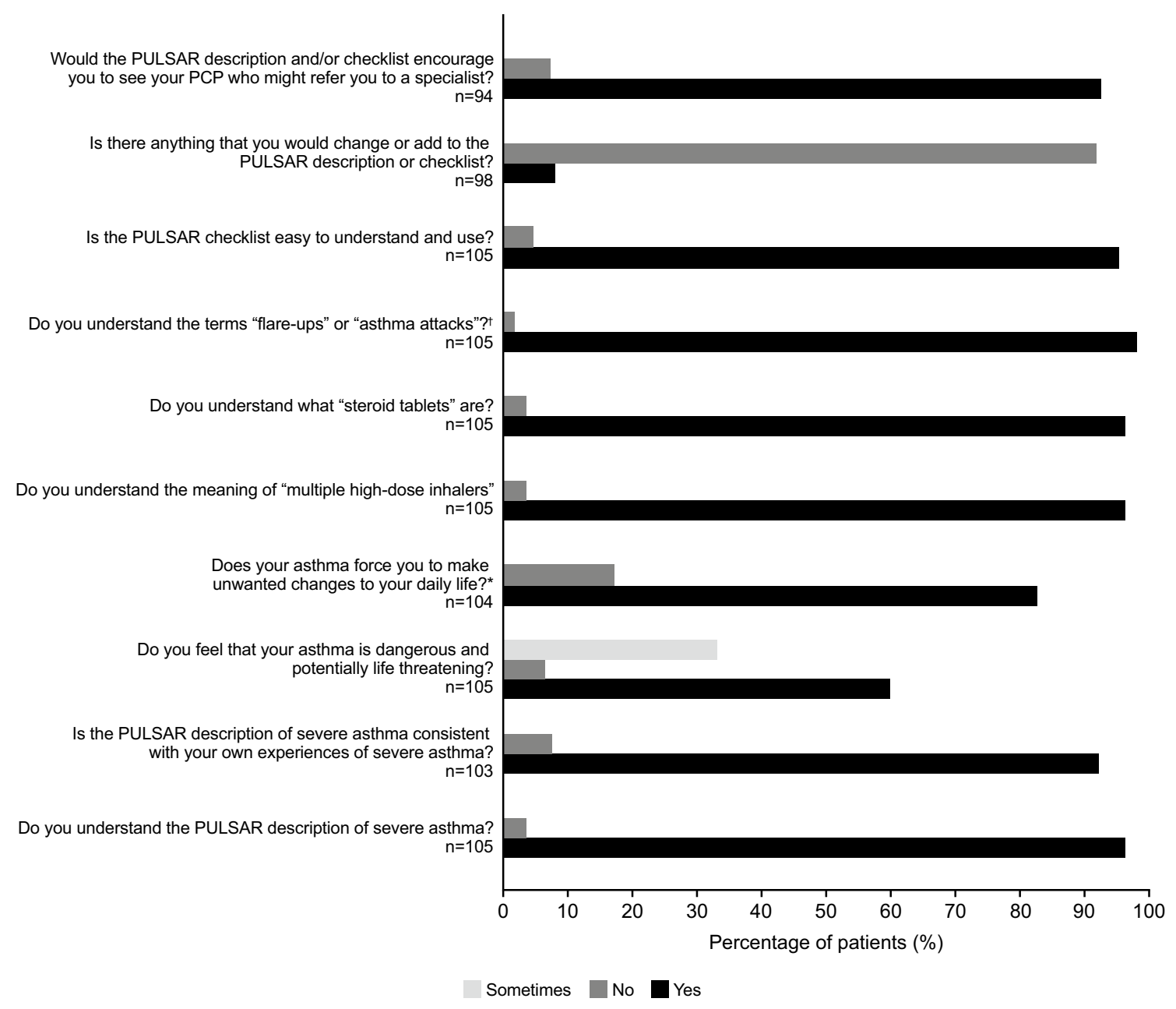

Fig. 1 The results of the patient survey. *Examples for unwanted changes to daily life provided in the survey include 'are you unable to do your normal day-today activities like household chores, meet with friends, work opportunities?' $68 / 105$ (64.7\%) patients preferred the term 'flare-ups' 


\section{The PULSAR description of severe asthma}

Severe asthma is a life-threatening condition. It can affect your entire life by forcing you to make unwanted changes to your daily activities. If you have severe asthma, you will experience frequent and severe worsening of asthma symptoms (asthma attacks/flare-ups). This will happen despite you taking multiple high-dose inhalers. These attacks or flare-ups often occur more than once per year. They will require steroid tablets or use of emergency services or hospitalization.

\section{What next?}

If you think you might have severe asthma, you should see your doctor. They may refer you to a specialist. A specialist may run some tests to see if you have severe asthma. If you have severe asthma, your doctor or specialist may be able to help you reduce your symptoms and improve your life.

Fig. 2 The final Patient Understanding Leading to Assessment for a Severe Asthma Referral (PULSAR) description of severe asthma

\section{Discussion}

As asthma is associated with debilitating symptoms that can affect HRQoL [1-3, 33], patients with severe asthma may benefit from a referral to a specialist to identify the most appropriate treatment options [26]. However, if patients are unaware that they have severe asthma they do not visit their PCP and receive the appropriate level of care. Therefore, a novel patient-centered description and accompanying checklist were developed to help patients understand and assess whether they might have severe asthma with the aim of encouraging patients to visit their PCP, who may refer them to a specialist, if appropriate. The final PULSAR description and patient checklist are included in Figs. 2 and 3 , respectively. According to the feedback received from a behavioral scientist and patients with severe asthma, the PULSAR description and checklist are considered to be relevant to this patient group and are likely to effect a change in patient behavior. The patient survey also demonstrated
Fig. 3 The Patient Understanding Leading to Assessment for a Severe Asthma Referral (PULSAR) checklist for patients to assess whether they might have severe asthma

\section{Do you have severe asthma?}

Please consider the Clear (Red) and Concerning (Yellow) signs of severe asthma in the checklist below. Please put a " $X$ " in the box next to any of the signs that apply to you. If any of the Clear (Red) signs apply to you or if you have put an " $X$ " next to some of the Concerning (Yellow) signs and you are worried, please visit your doctor and share this information. The goal is to help you reduce your symptoms and improve your quality of life.

\section{Severe asthma checklist:}

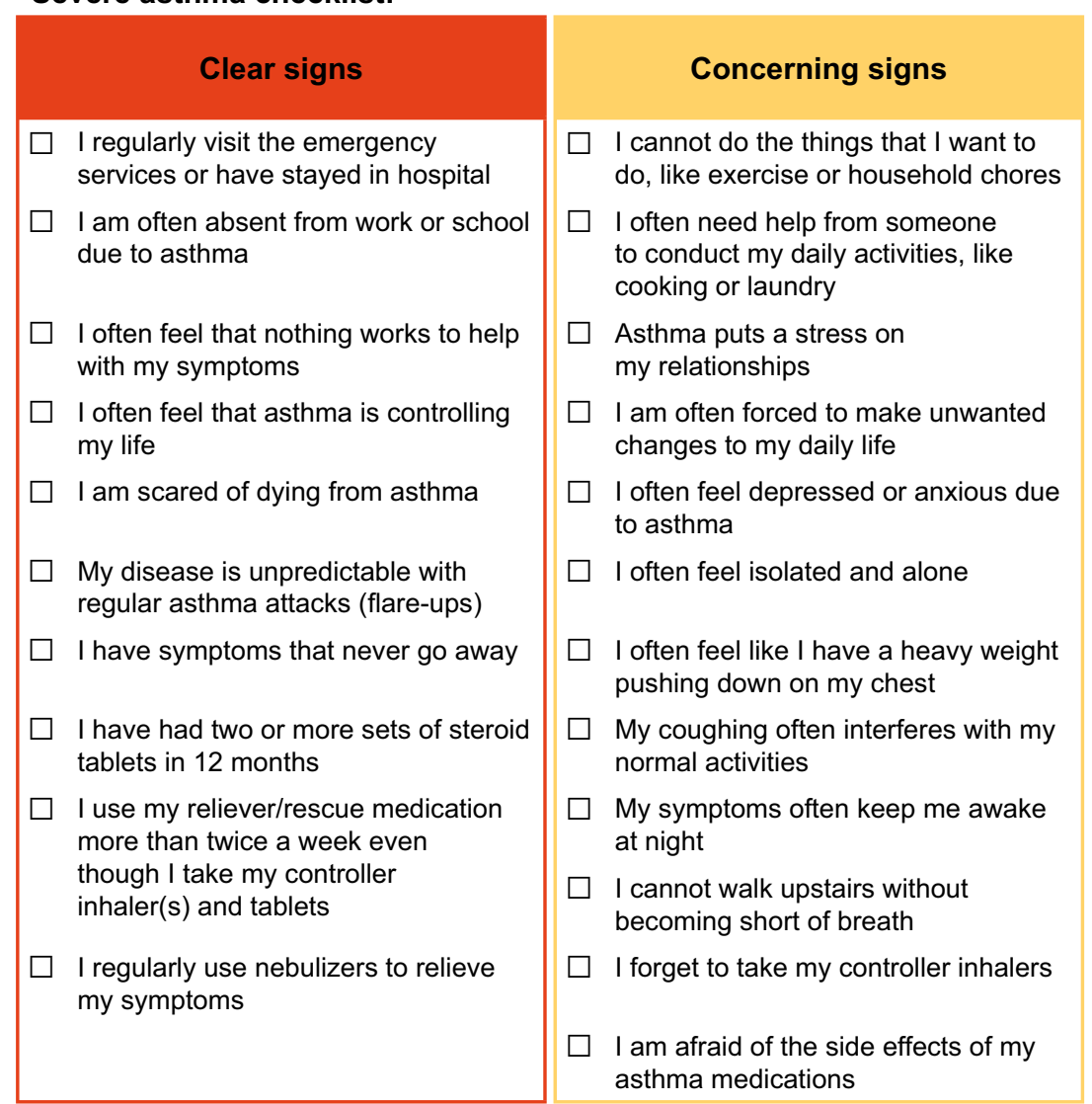


that no further changes to the PULSAR description and checklist were required and both items fulfilled the PULSAR initiative.

The definition of severe asthma published in the 2019 GINA pocket guide for difficult-to-treat and severe asthma describes severe asthma as "asthma that is uncontrolled despite adherence with maximal optimized therapy (GINA Step 4 or 5) and treatment of contributory factors (such as inhaler technique), or that worsens when high-dose treatment is decreased" [3]. The GINA pocket guide also suggests that the severe asthma phenotype should be assessed for patients uncontrolled on high-dose ICS or maintenance OCS within a specialist clinic [3]. These assessments include tests for various biomarkers of type 2 airway inflammation, including blood eosinophil counts $\geq 150$ cells $/ \mu \mathrm{L}$, sputum eosinophil counts $\geq 2 \%$, and/or fractional exhaled nitric oxide (FeNO) $\geq 20 \mathrm{ppb}$, as well as determining whether their asthma is allergy driven. This definition of severe asthma in GINA is in agreement with the definition from the International (ERS/ATS) Guidelines on Severe Asthma that have been almost universally accepted by the respiratory community [22]. Although the PULSAR description of severe asthma is largely consistent with this medical definition, it is simpler in its language to aid understanding and broader in its scope, including patient-centered concepts to capture their experience of this disease and to provide prompts to the patient to visit their doctor. Indeed, establishing a definition of severe asthma that patients can understand and use is of importance as various national initiatives worldwide allow patients to take more ownership in their own care. For example, in the US, since this working group was convened, patients now have access to their electronic medical records. Additionally, guidance from the National Institute for Health and Care Excellence (NICE) in the UK for the use of objective testing to confirm a diagnosis may drive more referrals [34].

There have been many studies that support the findings of the working group and the concepts and items chosen by the working group for inclusion in the PULSAR description and checklist, respectively. Previous studies have demonstrated that severe asthma can affect a patient's entire life, forcing them to make lifestyle compromises, consistent with the findings of the working group [35-39]. The European Fighting for Breath survey of severe asthma estimated that more than 6 million people live in fear that their next attack will be fatal and many restrict their physical activities or do not participate in social activities [38]. Supporting this, another study from the UK found that most (22/25) patients with severe asthma do not meet the NICE recommended levels of physical activity, mostly due to symptoms [36]. Furthermore, according to an Australian interview study and an Asthma UK survey, severe asthma significantly hinders patient lives, from daily chores to career, relationships, and family life [35, 39]. Similar to the checklist item on reliever/ rescue medication use, a previous study in Canada has shown that many patients do not follow the recommended regimen for their reliever/rescue medications [37]. In this Canadian study of 343,520 individuals with asthma contributing to 2.6 million person-years of data, 190,364 (7.3\%) person-years were categorized as inappropriate reliever users and $24,017(0.9 \%)$ as excessive users of reliever medications [37]. Consistent with the checklist item on the treatment side effects associated with steroid tablets, a previous study has demonstrated that patients have reported concerns about OCS side effects [35]. Similarly, a questionnaire-based study including participants attending an asthma awareness day in Norwich, UK, demonstrated that $82 \%$ of patients said that they disliked taking oral steroids and $12 \%$ said that they refuse to take them [40]. Weight gain was the most frequent concern, which was reported by $58 \%$ of patients [40]. Overall, previous studies support the inclusion of concepts and items within the PULSAR description and checklist, respectively.

The potential for patient-centered prompts to impact on clinical practice was recently demonstrated in the UK. Following a national campaign designed to raise awareness of persistent cough as a possible sign of lung cancer among the general public, urgent PCP referrals for suspected lung cancer and lung cancer diagnoses were seen to significantly increase compared with the same time in the previous year [41]. Furthermore, there was a significant increase in the number of surgical resections during the campaign period. Therefore, this new patient-centered description of severe asthma may improve clinical practice by increasing the number of appropriate referrals to a respiratory specialist, with a more informed patient. Increased referrals of appropriate patients will ensure that a greater number of patients with severe asthma receive the appropriate level of care, improving their overall health status (physical and mental) and HRQoL. However, although many of the asthma guidelines provide guidance on when patients should be referred to a specialist $[2,3,19,21,23]$, these guidelines are not always implemented [26]. Many asthma guidelines tend to differ in their recommendations, which may lead to confusion among HCPs on the most appropriate management strategies in severe asthma. In these cases, it is recommended that HCPs familiarize themselves with common patient terminology and behaviors so that they can refer patients to a specialist, when appropriate. GINA has recently provided guidance on when to refer patients with severe asthma based on treatment response and symptomatic control following the correction/ treatment of several risk factors [3]; however, additional HCP training may still be required [42].

Strengths of the PULSAR initiative and description include its multidisciplinary approach, robust methodology, and patient input. The working group included 
representatives from different backgrounds, which was key to developing a valid and acceptable definition. In addition, the PULSAR description underwent a thorough review process with these individuals, including a survey with patients with severe asthma. Although the PULSAR description is limited to English-speaking countries, the description will be adapted into the other 45 languages of the Global Allergy Asthma Patient Platform (GAAPP) member countries with rigorous local language review to improve its global application. The PULSAR description and checklist will be made available through the GAAPP website in the first instance, and then via patient group websites. Other possible uses in the future could include pamphlets in Emergency Rooms/Accident and Emergency departments or through online social media platforms.

There are some potential limitations of the initiative that should be discussed. Firstly, the PULSAR description and checklist were developed by a working group that included patients with severe asthma, and the subsequent survey was conducted in patients with severe asthma only. The exclusion of patients without severe asthma may have impacted the specificity of the PULSAR description of severe asthma. Secondly, limiting symptoms in the description to those that are severe does not rule out patients with mild asthma who may have severe symptoms during exacerbations. However, as the aim of the PULSAR initiative was to increase appropriate referrals by providing all patients with asthma with a prompt to go and see their doctor about their symptoms, as opposed to developing a validated patient-reported outcome for severe asthma, it is unlikely that these limitations will detract from the usefulness of the description and checklist to patients.

A third potential limitation was the fact that racial/ethnic and geographic data were not collected in the survey. Studies in patients with asthma have shown that patient outcomes are influenced by access to care, and that access to care varies according to ethnic/racial background [15]. Unfortunately, data on the ethnicity of the survey participants were not available, limiting our ability to determine what different groups of people thought about the PULSAR description and checklist. Another limitation relates to the design of the survey questions and the possible discordance between a patient's stated understanding of a given concept and the actual meaning of that concept, or between one patient's understanding and that of another. For example, if the patient answered 'Yes' when asked 'Do you understand what $x$ means?', their understanding was not subsequently checked and confirmed as being correct. However, the survey was designed to provide a simple 'sense check' of the PULSAR description and checklist, as opposed to a formal validation. Finally, although the PULSAR description and checklist were reviewed by an individual behavioral scientist, this could have introduced a degree of bias and may have benefitted from a wider range of views.

\section{Conclusion}

A novel and patient-centered description has been developed to improve patient understanding of severe asthma. The PULSAR description could help patients assess if they have severe asthma, prompting them to visit their PCP, and increase the number of appropriate referrals to a specialist.

Acknowledgements The authors would like to acknowledge the working group members who did not author this article, including Leonard Bacharier (Washington University in St Louis, MO, USA), Vanessa Foran (Asthma Canada, Canada), Vikki Knowles (NHS Guildford and Waverley Clinical Commissioning Group, Guildford, UK), Neil Martin (GSK, Brentford, UK/Glenfield Hospital in Leicester, Leicester, UK), Donna Matlach (US patient representative), Susanna Palkonen (European Federation of Asthma, Brussels, Belgium), Charlene Prazma (GSK, NC, USA), and Christine Rolland (Association Asthme \& Allergies, Boulogne, France). In addition, the authors acknowledge Dr Maureen George (behavioral scientist and certified asthma educator) from the Columbia University School of Nursing in New York, NY, USA, for her analysis of the description and, Cara Kraft (Allergy Asthma Network) for implementing the online polling questionnaire and for collating and analyzing the data. Editorial support in the form of initial preparation of the outline of this manuscript, including the patient-centric description, based on input from all authors, and collation and incorporation of author feedback to develop subsequent drafts, assembling tables and figures, copyediting, and referencing was provided by Rachael Baylie, PhD, of Fishawack Indicia Ltd, UK (as a direct in-kind agency support grant from GSK and additional funding by Global Allergy Asthma Patient Platform [GAAPP], a not-for-profit network representing over 40 nonprofit patient organizations worldwide [registered in Austria]).

Author contributions The authors met the criteria for authorship as recommended by the International Committee of Medical Journal Editors. TAW contributed to the conception and design of this initiative. All authors contributed to data interpretation, were involved in preparation and review of the manuscript, approved the final version to be submitted, and agree to be accountable for all aspects of the work.

\section{Compliance with Ethical Standards}

Funding The PULSAR working group (including the assessment of current severe asthma definitions, travel and accommodation costs, and honoraria for the attendees) was funded by a direct in-kind agency support grant from GSK. Neither the working group members nor authors received any direct compensation related to the development of this manuscript. Editorial support was funded by GSK, through a direct in-kind agency support grant, and Global Allergy Asthma Patient Platform (GAAPP), a not-for-profit network representing over 40 nonprofit patient organizations worldwide (registered in Austria).

Conflict of interest TAW is the elected president (voluntary position) of the Global Allergy Asthma Patient Platform, which is a charity. MJF is an employee of GSK, owns stocks/shares in GSK, and is an honorary research fellow at Asthma UK Centre for Applied Research, The University of Edinburgh. AM is a patient advisor for Asthma 
UK Centre for Applied Research and receives travel and accommodation expenses from AstraZeneca for a voluntary role in a Patient Partnership Program. DBP is an employee of Observational and Pragmatic Research Institute, Singapore, Singapore and Optimum Patient Care Ltd, Oakington, Cambridge, UK. DBP has board membership with Aerocrine, Amgen, AstraZeneca, Boehringer Ingelheim, Chiesi, Mylan, Mundipharma, Napp, Novartis, Regeneron Pharmaceuticals, Sanofi Genzyme, Teva Pharmaceuticals; consultancy agreements with Almirall, Amgen, AstraZeneca, Boehringer Ingelheim, Chiesi, GSK, Mylan, Mundipharma, Napp, Novartis, Pfizer, Teva Pharmaceuticals, Theravance; grants and unrestricted funding for investigator-initiated studies (conducted through Observational and Pragmatic Research Institute Pte Ltd) from Aerocrine, AKL Research and Development Ltd, AstraZeneca, Boehringer Ingelheim, British Lung Foundation, Chiesi, Mylan, Mundipharma, Napp, Novartis, Pfizer, Regeneron Pharmaceuticals, Respiratory Effectiveness Group, Sanofi Genzyme, Teva Pharmaceuticals, Theravance, UK National Health Service, Zentiva (Sanofi Generics); payment for lectures/speaking engagements from Almirall, AstraZeneca, Boehringer Ingelheim, Chiesi, Cipla, GSK, Kyorin, Mylan, Merck, Mundipharma, Novartis, Pfizer, Regeneron Pharmaceuticals, Sanofi Genzyme, Skyepharma, Teva Pharmaceuticals; payment for manuscript preparation from Mundipharma, Teva Pharmaceuticals; payment for the development of educational materials from Mundipharma, Novartis; payment for travel/accommodation/ meeting expenses from Aerocrine, AstraZeneca, Boehringer Ingelheim, Mundipharma, Napp, Novartis, Teva Pharmaceuticals; funding for patient enrollment or completion of research from Chiesi, Novartis, Teva Pharmaceuticals, Zentiva (Sanofi Generics); stock/stock options from AKL Research and Development Ltd, which produces phytopharmaceuticals; owns $74 \%$ of the social enterprise Optimum Patient Care Ltd (Australia and UK) and 74\% of Observational and Pragmatic Research Institute Pte Ltd (Singapore); and is a peer reviewer for grant committees of the Efficacy and Mechanism Evaluation programme, and Health Technology Assessment. AMW is an employee at the University of East Anglia and Norfolk and Norwich University Hospital, Norwich, UK. All authors received honoraria and travel costs for attending the PULSAR working group meeting.

Ethical approval For the survey used, formal consent was not required. Survey responses were provided anonymously, and all patients gave online consent for the results to be shared and included in publications.

Data availability The datasets generated during and/or analyzed during the current study are available from the corresponding author on reasonable request.

Open Access This article is distributed under the terms of the Creative Commons Attribution-NonCommercial 4.0 International License (http://creativecommons.org/licenses/by-nc/4.0/), which permits any noncommercial use, distribution, and reproduction in any medium, provided you give appropriate credit to the original author(s) and the source, provide a link to the Creative Commons license, and indicate if changes were made.

\section{References}

1. Bahadori K, et al. Economic burden of asthma: a systematic review. BMC Pulm Med. 2009;9:24.

2. Global Initiative for Asthma. Global Strategy for Asthma Management and Prevention. 2018. Available from: https://ginasthma. org/. Accessed 18 Apr 2018.
3. Global Initiative of Asthma. Difficult-to-treat and severe asthma in adolescents and adult patients: diagnosis and management, a GINA pocket guide for health professionals. 2019. Available from: https://ginasthma.org/wp-content/uploads/2019/04/GINA-Sever e-asthma-Pocket-Guide-v2.0-wms-1.pdf. Accessed 14 May 2019.

4. Food Allergy Canada. 2018. Available from: http://foodallerg ycanada.ca/. Accessed 18 Apr 2018.

5. British Lung Foundation. 2018. Available from: https://www.blf. org.uk/. Accessed 18 Apr 2018.

6. Allergy New Zealand. 2018. Available from: http://www.aller gy.org.nz/. Accessed 18 Apr 2018.

7. Canadian Network for Respiratory Care. 2018. Available from: http://cnrchome.net/. Accessed 18 Apr 2018.

8. Asthma Society of Canada. 2018. Available from: http://www. asthma.ca/napa/. Accessed 18 Apr 2018.

9. Asthma Society of Ireland. 2018. Available from: http://www. asthma.ie. Accessed 18 Apr 2018.

10. Allergy UK. 2018. Available from: https://www.allergyuk.org/. Accessed 18 Apr 2018.

11. Global Allergy and Asthma Patient Platform. 2018. Available from: https://gaapp.org/severe-asthma/. Accessed 18 Apr 2018.

12. Asthma UK. 2018. Available from: http://www.asthma.org.uk. Accessed 18 Apr 2018.

13. Asthma Australia/National Asthma Council (Australia). 2018. Available from: http://www.allergyfacts.org.au, http://www. nationalasthma.org.au. Accessed 18 Apr 2018.

14. The Asthma and Allergy Foundation of America. 2018. Available from: http://www.aafa.org/page/asthma-diagnosis.aspx. Accessed 18 Apr 2018.

15. Shields AE, Comstock C, Weiss KB. Variations in asthma care by race/ethnicity among children enrolled in a state Medicaid program. Pediatrics. 2004;113(3 Pt 1):496-504.

16. The Severe Asthma Foundation. 2018. Available from: https:// severeasthmafoundation.com/. Accessed 18 Apr 2018.

17. Allergy and Asthma Network. 2018. Available from: http:// www.allergyasthmanetwork.org. Accessed 18 Apr 2018.

18. Partridge MR, et al. Attitudes and actions of asthma patients on regular maintenance therapy: the INSPIRE study. BMC Pulm Med. 2006;6:13.

19. Asthma Canada. Severe asthma. 2017. Available from: https:// www.asthma.ca/wp-content/uploads/2017/06/What-is-Asthm a-Severe-Asthma.pdf. Accessed 18 Apr 2018.

20. Bousquet J, et al. Uniform definition of asthma severity, control, and exacerbations: document presented for the World Health Organization consultation on severe asthma. J Allergy Clin Immunol. 2010;126(5):926-38.

21. British Thoracic Society/Scottish Intercollegiate Guidelines Network. British guideline on the management of asthma, a national clinical guideline. 2016. Available from: https://www. brit-thoracic.org.uk/quality-improvement/guidelines/asthma/. Accessed 18 Apr 2018.

22. Chung KF, et al. International ERS/ATS guidelines on definition, evaluation and treatment of severe asthma. Eur Respir J. 2014;43(2):343-73.

23. Ichinose M, et al. Japanese guidelines for adult asthma 2017. Allergol Int. 2017;66(2):163-89.

24. National Asthma Council Australia. Austrailian asthma handbook. 2018. Available from: http://www.asthmahandbook.org. au/. Accessed 18 Apr 2018.

25. National Heart Lung and Blood Institute (US) and National Institutes of Health. National asthma education and prevention program. Third expert panel on the diagnosis and management of asthma. Expert panel report 3: guidelines for the diagnosis and management of asthma. 2007. Available from: https://www. nhlbi.nih.gov/files/docs/guidelines/asthgdln.pdf. Accessed 18 Apr 2018. 
26. Price D, et al. Asthma referrals: a key component of asthma management that needs to be addressed. J Asthma Allergy. 2017;10:209-23.

27. The National Asthma Council Australia. 2018. Available from: https://www.nationalasthma.org.au/. Accessed 18 Apr 2018.

28. GlaxoSmithKline. Advair Diskus patient information. 2019. Available from: https://www.gsksource.com/pharma/content/ dam/GlaxoSmithKline/US/en/Prescribing_Information/Advai r_Diskus/pdf/ADVAIR-DISKUS-PIL-IFU.PDF. Accessed 16 May 2019.

29. Package leaflet: information for the patient, Fasenra $30 \mathrm{mg}$ solution for injection in pre-filled syringe, benralizumab. 2019. Available from: https://www.medicines.org.uk/emc/files/pil.8918.pdf. Accessed 16 May 2019.

30. Package leaflet: information for the user, Nucala ${ }^{\circledR} 100 \mathrm{mg}$ powder for solution for injection, mepolizumab. 2018. Available from: https://www.medicines.org.uk/emc/files/pil.1938.pdf. Accessed 19 May 2019.

31. GlaxoSmithKline. Nucala, highlights of prescribing information. 2017. Available from: https://www.gsksource.com/pharma/conte nt/dam/GlaxoSmithKline/US/en/Prescribing_Information/Nucal a/pdf/NUCALA-PI-PIL.PDF. Accessed 18 Apr 2018.

32. AstraZeneca. Fasenra, highligts of prescribing information. 2017; Available from: https://www.azpicentral.com/fasenra/fasen ra.pdf\#page $=1$. Accessed 18 Apr 2018.

33. Gelfand EW. The impact of asthma on the patient, the family and society. Adv Stud Med. 2008;8:57-63.

34. National Institute for Health and Care Excellence (NICE). NICE Guideline NG80. Guidance on asthma: diagnosis, monitoring and chronic asthma management. 2017. Available from: https://www. nice.org.uk/guidance/ng80. Accessed 16 May 2019.
35. Foster JM, et al. "I have lost in every facet of my life": the hidden burden of severe asthma. Eur Respir J. 2017;50(3):1700765.

36. Livingston R, et al. Physical activity levels in a severe asthma population. Eur Respir J. 2017;50(suppl 61):PA4701. https://doi. org/10.1183/1393003.congress-2017.PA4701.

37. Tavakoli $\mathrm{H}$, et al. Predictors of inappropriate and excessive use of reliever medications in asthma: a 16-year population-based study. BMC Pulm Med. 2018;18(1):33.

38. European Federation of Allergy and Airways Diseases Patients Associations. A European patient perspective on severe asthma: fighting for breath. 2012. Available from: http://www.efanet.org/ images/2012/07/Fighting_For_Breath1.pdf. Accessed 12 Dec 2018.

39. Asthma UK. 75\% of people with severe asthma need regular emergency care, new Asthma UK survey finds. 2018. Available from: https://www.asthma.org.uk/about/media/news/75-of-peopl e-with-severe-asthma-need-regular-emergency-care-new-asthm a-uk-survey-finds/. Accessed 10 Dec 2018.

40. Price $\mathrm{D}$, et al. Concerns and misconceptions regarding steroid therapy in asthma: findings and impact of a public meeting. Prim Care Respir J. 1998;6:43.

41. Ironmonger L, et al. An evaluation of the impact of large-scale interventions to raise public awareness of a lung cancer symptom. Br J Cancer. 2015;112(1):207-16.

42. National Institute for Health and Care Excellence (NICE). Health and social care directorate quality standards and indicators briefing paper. 2018. Available from: https://www.nice.org.uk/guida nce/qs25/documents/briefing-paper. Accessed 10 Dec 2018.

43. Pavord ID, et al. After asthma: redefining airways diseases. Lancet. 2018;391(10118):350-400. 